University of Wollongong

Research Online

Faculty of Social Sciences - Papers (Archive) Faculty of Arts, Social Sciences \& Humanities

2004

Path integration deficits during linear locomotion after human medial temporal lobectomy

John W. Philbeck

University of Wollongong, johnp@uow.edu.au

Marlene Behrmann

Carnegie Mellon University

Lucien Levy

George Washington University

Samuel J. Potolicchio

George Washington University

Anthony J. Caputy

George Washington University

Follow this and additional works at: https://ro.uow.edu.au/sspapers

Part of the Education Commons, and the Social and Behavioral Sciences Commons

Research Online is the open access institutional repository for the University of Wollongong. For further information contact the UOW Library: research-pubs@uow.edu.au 


\title{
Path integration deficits during linear locomotion after human medial temporal lobectomy
}

\begin{abstract}
Animal navigation studies have implicated structures in and around the hippocampal formation as crucial in performing path integration (a method of determining one's position by monitoring internally generated self-motion signals). Less is known about the role of these structures for human path integration. We tested path integration in patients who had undergone left or right medial temporal lobectomy as therapy for epilepsy. This procedure removed approximately $50 \%$ of the anterior portion of the hippocampus, as well as the amygdala and lateral temporal lobe. Participants attempted to walk without vision to a previously viewed target 2-6 m distant. Patients with right, but not left, hemisphere lesions exhibited both a decrease in the consistency of path integration and a systematic underregistration of linear displacement (and/or velocity) during walking. Moreover, the deficits were observable even when there were virtually no angular acceleration vestibular signals. The results suggest that structures in the medial temporal lobe participate in human path integration when individuals walk along linear paths and that this is so to a greater extent in right hemisphere structures than left. This information is relevant for future research investigating the neural substrates of navigation, not only in humans (e.g., functional neuroimaging and neuropsychological studies), but also in rodents and other animals.
\end{abstract}

\section{Keywords}

temporal, during, after, lobectomy, human, deficits, locomotion, integration, path, medial, linear

\section{Disciplines}

Education | Social and Behavioral Sciences

\section{Publication Details}

Philbeck, J. W., Behrmann, M., Levy, L., Potolicchio, S. J. \& Caputy, A. J. (2004). Path integration deficits during linear locomotion after human medial temporal lobectomy. Journal of Cognitive Neuroscience, 16 (4), 510-520. 


\title{
Path Integration Deficits during Linear Locomotion after Human Medial Temporal Lobectomy
}

\author{
John W. Philbeck ${ }^{1}$, Marlene Behrmann ${ }^{2}$, Lucien Levy ${ }^{3}$, \\ Samuel J. Potolicchio ${ }^{3}$, and Anthony J. Caputy ${ }^{3}$
}

\begin{abstract}
Animal navigation studies have implicated structures in and around the hippocampal formation as crucial in performing path integration (a method of determining one's position by monitoring internally generated self-motion signals). Less is known about the role of these structures for human path integration. We tested path integration in patients who had undergone left or right medial temporal lobectomy as therapy for epilepsy. This procedure removed approximately $50 \%$ of the anterior portion of the hippocampus, as well as the amygdala and lateral temporal lobe. Participants attempted to walk without vision to a previously viewed target $2-6 \mathrm{~m}$ distant. Patients with right, but not left, hemisphere lesions exhibited
\end{abstract}

both a decrease in the consistency of path integration and a systematic underregistration of linear displacement (and/or velocity) during walking. Moreover, the deficits were observable even when there were virtually no angular acceleration vestibular signals. The results suggest that structures in the medial temporal lobe participate in human path integration when individuals walk along linear paths and that this is so to a greater extent in right hemisphere structures than left. This information is relevant for future research investigating the neural substrates of navigation, not only in humans (e.g., functional neuroimaging and neuropsychological studies), but also in rodents and other animals.

\section{INTRODUCTION}

An important function of vision is to facilitate navigation from one location to another. As important as this function is, however, visual information is frequently degraded or made unavailable by the common occurrences of occlusions and poor lighting conditions. This being the case, it is advantageous for sighted individuals to remain able to navigate without vision. Many animals have this ability (Etienne, Maurer, \& Séguinot, 1996; Wehner, Michel, \& Antonsen, 1996), and humans are no exception. The average human can sight a target up to $20 \mathrm{~m}$ away or more, and then walk to it quite accurately while blindfolded (for a review, see Mittelstaedt \& Mittelstaedt, 2001). The accuracy of this nonvisual navigation is evidence that the brain is exquisitely tuned to sense the self-motion signals generated by walking and to use them for the purpose of controlling behavior. The neural processes underlying nonvisual navigation remain poorly understood, however.

When navigating without vision, the sensory information for determining one's position is restricted to signals arising from the vestibular apparatus and signals related to muscular activity (e.g., proprioception and efference copy). The process of determining one's position on the basis of internally generated (idiothetic)

${ }^{1}$ The George Washington University, ${ }^{2}$ Carnegie Mellon University, ${ }^{3}$ The George Washington University Medical Center

self-motion signals is known as path integration or dead reckoning (Etienne et al., 1996). An updated estimate of one's current position may be maintained by integrating these self-motion signals over time. Path integration is formally distinct from other types of navigation in which one uses vision, or some other sensory modality, to determine one's position relative to environmental features ("landmarks") at known locations.

Effective path integration entails deriving a representation of the current displacement from one's last known position. There is abundant evidence that the medial temporal lobe (MTL) plays an important role in the processing of spatial information. The firing rate of pyramidal cells in the rodent hippocampus is highly correlated with the location of the animal in space (for a review of this extensive literature, see Redish, 1999). One interpretation of such a result is that these socalled "place cells" participate in representing the layout of the environment (O'Keefe \& Dostrovsky, 1971). Consistent with this view, rodents with lesions that damage the hippocampus or its connections tend to perform poorly on tasks such as the radial arm maze and water maze, which require the animal to demonstrate its memory for locations it has visited previously (e. g., Morris, Garrud, Rawlins, \& O'Keefe, 1982). Spatial memory deficits are manifested after MTL injury in monkeys as well, particularly after damage to the parahippocampal gyrus surrounding the hippocampus (Murray \& Mishkin, 1998). 
There is evidence that the MTL's role in navigation extends beyond simply representing spatial information to include the assimilation of idiothetic self-motion signals into existing spatial representations. Place cells, for example, continue to show location-dependent firing even when the animal's vision is occluded (Markus, Barnes, McNaughton, Gladden, \& Skaggs, 1994; Quirk, Muller, \& Kubie, 1990), suggesting that these cells participate in the processing of idiothetic self-motion signals. In the monkey, some hippocampal cells have been found to respond to whole-body motion (O'Mara, Rolls, Berthoz, \& Kesner, 1994). Several conceptualizations of hippocampal function suggest that the MTL's role in path integration (including processing and storing spatial, temporal, and self-motion information) emerges as part of a more general role for subserving episodic memory (e.g., Burgess, Maguire, \& O'Keefe, 2002; Vargha-Khadem et al., 1997), declarative memory (e.g., Squire, 1992), relational processing (e.g., Eichenbaum \& Cohen, 2001), or contextual processing (e.g., Redish, 2001).

Rodents and humans behave similarly in water-mazetype navigation tasks (Hamilton, Driscoll, \& Sutherland, 2002), but clearly humans use more sophisticated representations to control navigation than do rodents, at least under certain circumstances (e.g., Wang \& Spelke, 2002). The extent to which human navigation within the local environment parallels rodent (and monkey) navigation is still poorly understood, however. The need to understand the homology between rodent and human brain structures that participate in navigation is particularly pressing, given the enormous body of research that is currently available based on this animal model. A host of neuropsychological and functional neuroimaging studies support the view that MTL structures play a critical role in human navigation. Much work has implicated the right hippocampus and/or parahippocampal gyrus as important substrates for spatial memory in humans, particularly when information must be retained for more than several seconds and less than several minutes (Pierrot-Deseilligny, Müri, Rivaud-Pechoux, Gaymard, \& Ploner, 2002; Feigenbaum, Polkey, \& Morris, 1996; Owen, Milner, Petrides, \& Evans, 1996; Smith \& Milner, 1981). Furthermore, humans with MTL damage show deficits in tasks that require navigation to remembered locations in real and virtual environments (Astur, Taylor, Mamelak, Philpott, \& Sutherland, 2002; Spiers et al., 2001; Skelton, Bukash, Laurance, Thomas, \& Jacobs, 2000; Bohbot et al., 1998). Functional neuroimaging studies in neurologically intact humans have also found that the hippocampus or parahippocampal gyrus becomes activated as participants navigate in a simulated 3-D environment (Grön, Wunderlich, Spitzer, Tomczak, \& Riepe, 2000; Maguire et al., 1998; Aguirre, Detre, Alsop, \& D'Esposito, 1996).

Although these studies support the notion that MTL structures play an important role in spatial memory, much less research has focused directly on MTL participation in processing human idiothetic self-motion signals. Observing the behavior of patients with MTL damage in nonvisual locomotor navigation tasks is a powerful tool for investigating these issues. Walking generates strong idiothetic self-motion signals and creates a situation in which vestibular and muscular cues are mutually consistent, as they are during real-world navigation. The occlusion of vision during locomotion excludes landmark-based navigation, thereby providing a narrow experimental focus on idiothetic self-motion signals. If the MTL participates in processing such signals, MTL injury should result in path integration deficits.

The MTL does seem to be involved in these tasks, but important details of its role remain to be characterized. Patients with MTL lesions, particularly in the right hemisphere, show path integration deficits during wholebody rotations as well as in nonvisual walking tasks involving a combination of turns and straight segments (Worsley et al., 2001; Wiest, Müller, Glück, Deecke, \& Baumgartner, 2000). The ability of such patients to perform path integration along purely linear paths is less clear, however. Worsley et al. (2001) concluded that the path integration errors manifested by their patients were attributable to deficits in rotational updating only, but speculated that their methods may not have been sufficiently sensitive to detect deficits in updating along linear paths. One possible source of insensitivity stems from their use of unimodal distance and route reproduction tasks, in which participants are exposed to a stimulus path by walking and then must reproduce that path, again using the walking modality. If participants perform path integration similarly on the stimulus and response portions of the path, systematic errors in the two segments could cancel, thereby yielding a potentially misleading pattern of accurate performance. The possible impairment of linear path integration after MTL damage is important to verify using more sensitive methods because it stands to elucidate which sensory inputs to path integration are processed in the MTLs. In natural situations, rotating one's body to face another direction generates strong rotational vestibular signals and a relatively small amount of leg movement. Walking along linear paths, meanwhile, produces the opposite pattern. If path integration is impaired during both linear and rotational locomotion after MTL damage, this would indicate that the MTL's role in path integration encompasses signals from multiple sensory systems rather than primarily those arising from the vestibular apparatus.

Our primary goal in the following study was to characterize the effect of MTL injury on path integration along linear paths. A surgical procedure designed to treat MTL epilepsy provides a unique opportunity to investigate this issue. This procedure, involving a partial medial temporal lobectomy, removes approximately two thirds of the anterior hippocampus and the structures surrounding it on one side of the brain. The lesions are 
too large to allow a fine-grained assessment of localization of function, but nevertheless provide a means of addressing our primary goal of characterizing the path integration deficits that occur after MTL damage. The unilateral nature of the lesion also permits an analysis of a possible hemispheric specialization. This methodology, then, presents an exceptional opportunity to investigate the critical issue of the homology of function between rodent and human MTL structures.

We tested patients with unilateral right or left hemisphere temporal lobe resections (RTLR and LTLR, respectively) and neurologically intact control participants in four behavioral tasks. Two of these tasks evaluated path integration: In the "target-directed walking" task, participants attempted to walk without vision to a previewed target. This task provides a measure of path integration and spatial memory, as well as a nonverbal indication of perceived target distance (Philbeck \& Loomis, 1997). This is a multimodal task in that the stimulus distance is specified by vision and the response is indicated via walking. In the "experimenterguided walking" task, participants walked without vision under the guidance of an experimenter and then verbally estimated the path length. Thus, the stimulus and response phases again entailed different modalities-in this case, the stimulus was supplied by walking and the response involved verbal estimation. This task provides a separate measure of path integration without any reliance upon visual perception. Multimodal tasks are more sensitive than unimodal tasks to systematic errors in path integration because they mitigate against the possibility that errors incurred during the stimulus phase could be canceled by similar errors in the response phase. The remaining two tasks were designed to evaluate visual perception and spatial memory without drawing upon path integration ability, given that these two processes are also implicated in target-directed walking: In one of these tests, participants verbally estimated the egocentric (absolute) distance to a visible target, and in the other, they viewed one target and then attempted to position a second one such that it reproduced the distance of the first. The second target in the distance matching task was positioned after a short delay to verify that participants could remember the target location long enough to walk to it if required.

We predicted that all participants would be fairly accurate in the verbal distance estimation and delayed distance matching tasks, given the well-lit environment and short $(<20 \mathrm{sec})$ retention intervals involved (Pierrot-Deseilligny et al., 2002; Bohbot et al., 1998). However, if structures within the MTL play a role in path integration that encompasses both linear and rotational self-motion, the patients should show some impairment in the two tasks that require path integration along linear trajectories relative to control participants. Based on previous results using similar methods (Worsley et al.,
2001), we anticipated that deficits in the two walking tasks would be more pronounced in the right MTL patients than the left.

\section{RESULTS}

If tissue that plays an important role in path integration is damaged or removed, the remaining structures must operate on a more sparse set of inputs. This could have a variety of effects on signals processed within the residual network, such as systematically biasing the signals or decreasing the signal-to-noise ratio. We therefore set out to characterize path integration performance not only in terms of systematic tendencies to over- or underestimate the extent of self-motion (i.e., response bias), but also in terms of within-subject random error (i.e., response consistency). To obtain a more robust estimate of central tendency in each participant's data, outliers more than 4 standard deviations from the mean were removed in the experimenter-guided and targetdirected walking tasks. This resulted in the removal of approximately $5 \%, 4 \%$, and $3 \%$ of the data for the control, LTLR, and RTLR groups, respectively, and caused only trivial differences in the statistical analyses relative to tests performed on the full data set. To analyze withinsubject response consistency (i.e., random error), we used the least squares criterion to find the best-fitting straight line through each participant's responses. Although we did not test nonlinear models, these linear fits were generally excellent; across the four tasks, the mean squared correlation coefficients were $.91, .87$, and .87 for the control, LTLR and RTLR groups, respectively. We then took the standard error of estimate (SEE) of the best-fitting lines as a measure of overall consistency. We subjected the SEEs to an analysis of variance (ANOVA), with "group" included as a between-subjects variable (control, LTLR and RTLR). To analyze group differences in constant error (bias), we performed four ANOVAs on the signed response error scores, one for each condition, with stimulus distance $(2.5$ and $5.0 \mathrm{~m})$ included as a within-subject variable. Each participant's data were averaged across repetition before analysis.

\section{Visual Perception and Spatial Memory}

\section{Within-Subject Random Error (Consistency)}

There were no reliable group differences in response consistency in the verbal distance estimation task, as measured by an ANOVA performed on the SEEs, $F(2,25)=1.63 ;$ SEM $=.105 ; p=.216$. The mean SEEs for the control, LTLR and RTLR groups were .32, .38, and $.52 \mathrm{~m}$, respectively. There was a small but reliable effect of group in the SEEs of the delayed distance matching task, $F(2,25)=3.89 ;$ SEM $=.042 ; p=.034$. Pairwise planned contrasts $(p=.05)$ showed that this effect was due to differences between the RTLR and control groups (mean SEE: $.43 \mathrm{~m}$ vs. $.30 \mathrm{~m}$ for RTLR and control, 
respectively). The mean SEE for the LTLR group fell between those of the other two groups $(.38 \mathrm{~m})$, but did not differ reliably from either. Taken together, these data indicate that the RTLR procedure is associated with mild decreases in response consistency in tasks requiring visual perception and maintenance of a single target location in memory for fairly short durations (15-20 sec).

\section{Constant Error (Bias)}

In the verbal distance estimation condition, there was a slight tendency for the LTLR group to underestimate the target distance systematically relative to the other groups, but this tendency did not reach statistical significance (see Table 1 and Figure 1A). There were no group differences in constant error in the delayed distance matching condition and, in fact, the responses of the two patient groups were generally accurate (Figure 1B). Thus, the RTLR group's decreased response consistency in this task was not accompanied by systematic response biases. Taken together, the Distance Estimation and Distance Matching tests suggest that there were no striking systematic deficits in visual space

Table 1. Source Tables for Analyses of Variance of Mean Signed Responses

\begin{tabular}{lrrr}
\hline Condition & SEM & F ratio & p value \\
\hline Target-directed walking & & & \\
Group $(2,25)$ & 2.47 & 2.81 & .079 \\
Distance $(1,25)$ & 111.41 & 631.92 & $<.001$ \\
Group $\times$ Distance $(2,25)$ & .93 & 5.28 & .012
\end{tabular}

Experimenter-guided walking

Group (2, 25)

Distance $(1,25)$

Group $\times$ Distance $(2,25)$

3.60

.042

Verbal distance estimation

Group (2, 25)

Distance $(1,25)$

Group $\times$ Distance $(2,25)$

$$
3.02
$$

83.62

193.67

.73

1.70

.203

Delayed distance matching

Group (2, 25)

Distance $(1,25)$

Group $\times$ Distance $(2,25)$

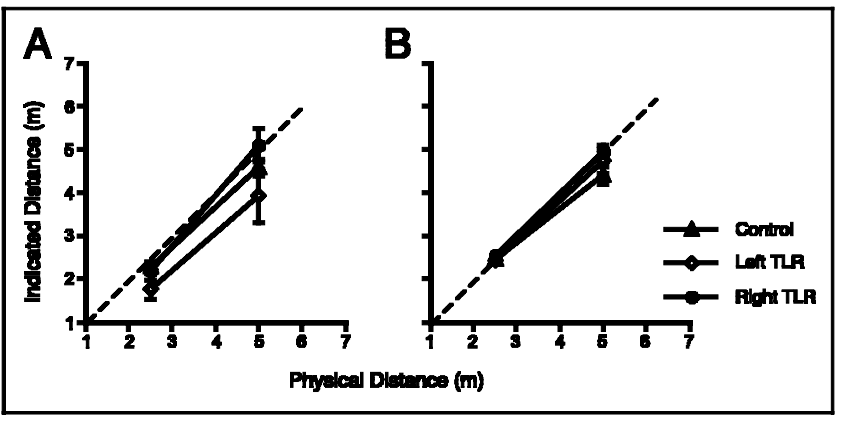

Figure 1. Average indicated distances for the three groups of participants (TLR $=$ temporal lobe resection). (A) Verbal distance estimation trials. (B) Delayed distance matching trials. Error bars denote \pm one standard error of the mean calculated across participants. The dashed line indicates accurate performance.

perception or in short-duration spatial memory in the patient groups, at least as measured by these tasks.

\section{Path Integration Tasks}

\section{Within-Subject Random Error (Consistency)}

There were no reliable group differences in the response consistency in the experimenter-guided walking condition, $F(2,25)=2.84$, SEM $=.241, p=.078$. The mean SEEs for the control, LTLR and RTLR groups were $.53, .33$, and $.66 \mathrm{~m}$, respectively. However, there was a group effect in the target-directed walking condition, $F(2,25)=5.04, S E M=.32, p=.014$. In particular, the responses of the RTLR patients were significantly less consistent than those of the other groups. Pairwise post hoc contrasts confirmed that this effect was primarily due to differences between the RTLR and control groups $(F=9.99 ; p=.004)$; no other contrasts reached significance. The mean SEE for the control group was $.28 \mathrm{~m}$, while the corresponding mean for the RTLR group was more than twice that $(.64 \mathrm{~m})$. The mean SEE for the LTLR group was intermediate $(.49 \mathrm{~m})$. Thus, one consequence of right MTL injury may be increased random error in path integration processes.

\section{Within-Subject Constant Error (Bias)}

The participants generally walked quite accurately in the target-directed walking condition, with the exception of the RTLR group (Figure 2A). Notably, the RTLR patients tended to overshoot when attempting to walk to previously viewed targets. This tendency became more pronounced for the 5-m target (Group $\times$ Distance interaction, Table 1), when the average overwalking exceeded $15 \%$ of the target distance. This interaction is to be expected if there are systematic biases in selfmotion sensing, as these errors would tend to accumulate as walked distance increases. Figure 3 shows the mean stopping locations for the individual participants in walking to the 5-m target; although the performance 
of several patients in the RTLR group fall within the range of normal performance, the distribution of the RTLR group is clearly shifted toward positive constant errors relative to the LTLR and control groups. Pairwise planned contrasts showed that the Group $\times$ Distance interaction was driven by differences between the RTLR responses and each of the other groups (RTLR vs. control: $F=8.43, p=.008$; RTLR vs. LTLR: $F=7.07, p=.013)$. The control and LTLR groups did not differ.

In the experimenter-guided walking condition, there was a general tendency toward underestimation of walked distances (approximately 30\% of the stimulus distance; see Figure 2B). The LTLR group's responses were somewhat more underestimated than the other groups', particularly at the 5-m stimulus distance (Group $\times$ Distance interaction, Table 1$)$. In this way, the LTLR group exhibited a similar tendency toward verbal underestimation as in the verbal distance estimation condition. Pairwise planned contrasts $(p=.05)$ showed that the interaction was driven by differences between the LTLR group and the other groups (LTLR vs. control: $F=6.13$, $p=.02$; LTLR vs. RTLR: $F=4.99, p=.035$ ). The control and RTLR groups did not differ from each other. This means that although the RTLR group showed the pattern that would be predicted for individuals who underestimate their self-motion (overshooting in target-directed walking trials and underestimating in experimenterguided walking trials), they only differed from normal behavior when walking to a previously seen target.

Estimates of walked distance in the experimenterguided walking trials were given verbally. At least some of the systematic bias in these trials, then, could be due to errors in the calibration of the verbal estimates. There were no reliable group differences in the verbal distance estimation trials, and the responses themselves were generally fairly accurate. Nevertheless, it may be that the analysis of the experimenter-guided walking data is less sensitive than it might be if the verbal

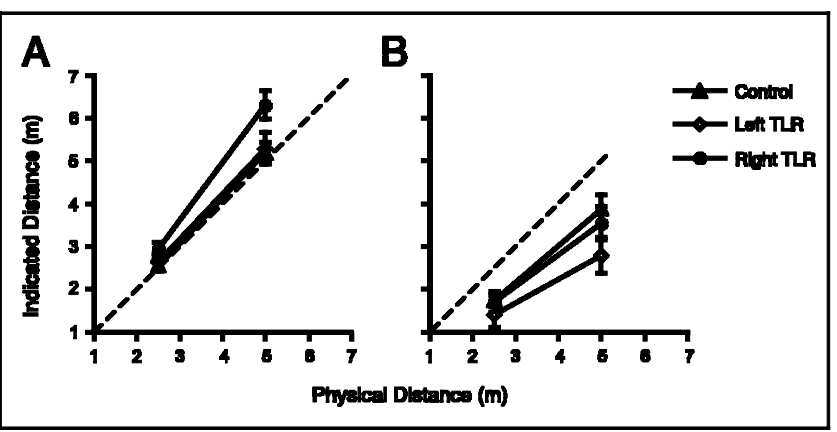

Figure 2. Average indicated distances for the three groups of participants (TLR $=$ temporal lobe resection). (A) Target-directed walking trials. (B) Experimenter-guided walking trials. Error bars denote \pm one standard error of the mean calculated across participants. The dashed line indicates accurate performance.

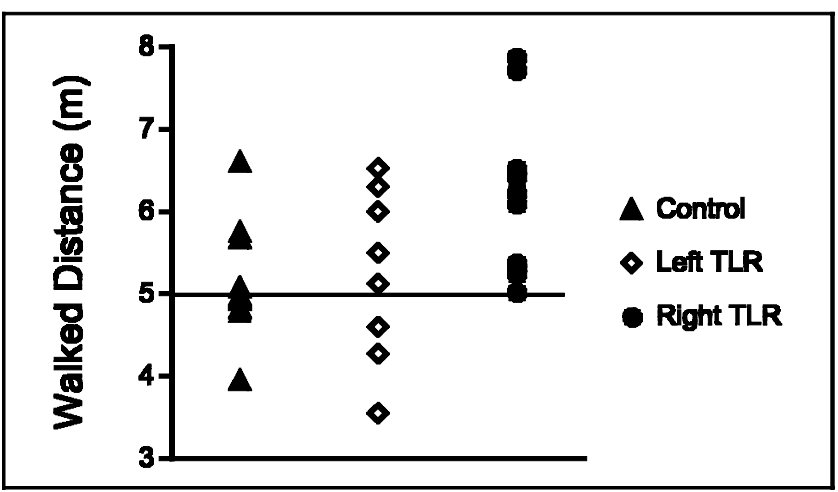

Figure 3. Average walked distances for a target at $5 \mathrm{~m}$ (target-directed walking trials). Each data point is the mean response for one participant, collapsed over five or six measurements; data for all three participant groups are shown (TLR $=$ temporal lobe resection). The solid horizontal line indicates accurate performance.

calibration for each participant were known with more certainty. Preliminary attempts to correct for verbal calibration errors in the experimenter-guided walking trials based on performance in the verbal distance estimation trials did not show any striking differences from the results presented in Table 1, so we did not pursue analyses of this kind further.

\section{Absolute (Unsigned) Error}

Analyses of signed error can underestimate systematic bias to the extent that positive and negative errors tend to cancel each other out. Separate ANOVAs were therefore performed on the absolute errors in the four experimental conditions. The outcome of these analyses generally mirrored those of the signed error tests, with the following exceptions. In target-directed walking trials, there were no differences between groups, $F(2,25)=2.052 ; p=.1496$, and no Group $\times$ Distance interaction, $F(2,25)=2.666 ; p=.089$. Taken in conjunction with the signed error analysis, this suggests that the effect of right hemisphere MTL lesions on targetdirected walking is primarily manifested in terms of overshooting rather than overall error. There was a main effect of group in the experimenter-guided walking condition, $F(2,25)=3.725 ; p=.0384$. Pairwise planned contracts $(p=.05)$ suggested that this was attributable to a larger amount of overall error in the LTLR group than in the other two groups, which did not differ from each other. The LTLR groups' error was primarily due to their underestimations in this condition, a result that is noticeable in Figure 2B.

\section{DISCUSSION}

As a group, right hemisphere medial temporal lobectomy patients tended to overshoot when attempting to walk without vision directly to previously seen targets. 
By contrast, left medial temporal lobectomy patients and neurologically intact participants arrived at the target location more accurately. The overshooting exhibited by the RTLR group was accompanied by a twofold increase in the within-subject variability of walking responses relative to the control participants. When no locomotion was required, the RTLR patients were able accurately to match target distances after a delay (albeit with somewhat decreased consistency) and performed well when verbally judging the distance to visual targets. Good performance on these tasks indicates that neither misperception of the target's initial position nor general deficits in short-term spatial memory could account for the systematic tendency to overwalk. Notably, the RTLR patients tended to verbally underestimate walked distances when they were guided by an experimenter. Although their performance did not differ from the control group's in this task, a point to which we will return below, the complementary pattern of overshooting in one task and underestimation in the other shows that there was no general tendency to produce too large or too small responses as a result of right MTL injury. Instead, we interpret this pattern as resulting from a systematic underperception of the extent of self-motion in both tasks. We conclude that structures within the right hemisphere MTL do indeed participate in path integration along linear trajectories.

The LTLR group did not differ from neurologically intact control participants in terms of the accuracy and consistency of target-directed walking while blindfolded. This good performance indicates that the LTLR group was able accurately to perceive and remember the target location. Their good performance on the Delayed Matching task corroborates this conclusion. In sum, there appears to be some lateralization of function of human path integration processes; although our work does not rule out left hemisphere involvement, right hemisphere structures apparently play the more crucial role. This conclusion accords well with the results of Worsley et al. (2001), and extends that previous work by confirming that RTLR surgery disrupts path integration along linear paths as well as paths that contain whole-body rotations. In addition, we have shown that right MTL injury is associated with not only systematic biases in path integration along linear paths but also decreases in response consistency. Importantly, the biases in path integration associated with RTLR are manifested as errors of underestimation for both linear locomotion (as shown in our study) and whole-body rotations (Worsley et al., 2001; Wiest et al., 2000).

Interestingly, whereas the RTLR participants showed evidence of underestimating self-motion in both targetdirected walking and experimenter-guided walking tasks, the neurologically intact control group responded accurately in the former task but underesti- mated in the latter. Both the RTLR and control groups accurately estimated the distance to visual targets when no locomotion was required, so apparently their underestimations in the experimenter-guided task were not simply a result of poor verbal calibration. Previous work has shown that path integration can be greatly enhanced when an individual has prior information about the magnitude of an upcoming trajectory before walking begins (Philbeck, Klatzky, Behrmann, Loomis, \& Goodridge, 2001). Vision of the target supplies this information in target-directed walking trials, but there is no such prior knowledge in the experimenter-guided walking task because participants do not know how far they will be guided on each trial. Thus, the control group may have in fact underestimated the extent of their self-motion in experimenter-guided trials but perceived this motion more accurately in target-directed walking trials. It may be that the RTLR surgery interferes with the benefit that is usually afforded to neurologically intact individuals when vision of a target is available before blindfolded walking begins. One possible account is that vision of a target and the anticipation of walking to it forms the basis of a spatiotemporal context for locomotion. When circumstances allow healthy individuals to establish such a contextual framework, it acts to facilitate the integration of self-motion information during the walk; after right MTL injury, however, this benefit does not materialize because processing of contextual and/or relational information is impaired (Eichenbaum \& Cohen, 2001; Redish, 2001).

Although our results suggest that RTLR patients show path integration deficits in linear paths, Worsley et al. (2001) did not find evidence of these errors in either of their two groups of patients with MTL resections. Although this apparent discrepancy might be due to superficial methodological differences between our two studies (e.g., our manipulation of walking speed vs. their use of vocal number repetition to discourage pace counting), a more likely explanation is that the multimodal reproduction tasks used here are more sensitive to path integration errors than are unimodal tasks. Interestingly, Worsley et al. also found no evidence of deficits in linear path integration in their homing vector task, in which blindfolded participants were guided along two legs of a triangle and then attempted to return directly to the origin of locomotion. The authors used a variety of stimulus trajectories, with the ideal response for each path entailing the creation of a final straight segment of $2.5 \mathrm{~m}$. Triangle completion is an important tool for characterizing human path integration (and indeed, Worsley et al. found that RTLR patients were impaired in rotational path integration in this task); however, its sensitivity for detecting path integration deficits along linear trajectories may be limited when there is little variation in the required response path length. Testing a broader range 
of required response path lengths promises to increase the sensitivity of future studies involving MTL patients and to enhance efforts toward computational modeling of human path integration (e.g., Klatzky, Beall, Loomis, Golledge, \& Philbeck, 1999).

We have used the term MTL advisedly throughout this paper in that we cannot localize more precisely the neural region in these patients that mediates the path integration deficit. The surgical resection includes portions of the hippocampus, subicular complex and amygdala, parts of the perirhinal and entorhinal cortices, the parahippocampal gyrus, and the anterolateral temporal lobe. In monkeys, the anterolateral temporal lobe and amygdala appear to be specialized for object recognition and emotional processing and are not likely to play a role in path integration (Logothetis \& Sheinberg, 1996; Sutherland \& McDonald, 1990). In rodents, the role of the hippocampus proper for path integration has been vigorously debated (for recent reviews, see Redish, 2001; Whishaw, Hines, \& Wallace, 2001), but there is general agreement that either the hippocampus or structures surrounding it (e.g., the subicular complex or entorhinal cortex) are key. Nevertheless, more research is needed to characterize the individual contribution of the other excised structures, as well as spared extratemporal tissue, toward the various processes underlying path integration.

We should also note that although we have argued for a hemispheric difference between the RTLR and LTLR groups, another, albeit unlikely, interpretation concerns the fact that the LTLR group was tested somewhat later after surgery than the RTLR group. This difference leaves open the possibility that systematic biases in path integration may be associated with unilateral damage to either left or right MTL structures; in this view, other brain regions may gradually assume some of the path integration functionality. Although possible, this interpretation is unlikely in view of the hemispheric differences in path integration evinced by the age-matched patient groups described by Worsley et al. (2001). Similarly, other deficits in spatial processing (e.g., spatial memory) are known to be more strongly associated with right rather than left hemisphere MTL injury (Nunn, Graydon, Polkey, \& Morris, 1999; Smith \& Milner, 1981).

Given that effective path integration relies in part upon the integrity of spatial memory, can the deficits observed in the RTLR group be attributed solely to memory impairments? Our Delayed Matching and Target Directed Walking tasks involved similar retention intervals, but they did not yield similar differences between the RTLR and control groups. There were only small differences in response consistency in Delayed Matching, whereas there were larger differences in both response consistency and bias in the walking task. Although this suggests that impairments in memory do not completely account for the RTLR group's path integration performance, more testing is required to resolve this issue. The possibility remains that the observed deficits are the result of more global impairments in relational, temporal, or contextual processing.

The critical result from this study is the evidence of deficits in human path integration along linear paths associated with right hemisphere MTL damage. Rodent studies have confirmed that MTL structures are implicated in navigation, but this is the first evidence of MTL involvement in path integration along purely linear paths in either humans or other animals. Although further research is needed to specify the relative importance of specific structures within the MTL in producing the deficits, we have shown that the deficits are manifested as both a decrease in the consistency of path integration and also as a systematic underregistration of linear displacement (and/or velocity) during nonvisual walking. Moreover, the deficits are observable even when there are virtually no angular acceleration signals coming from the vestibular apparatus. These findings not only clarify the conditions under which path integration deficits can occur after unilateral medial temporal lobectomy, but also the nature of those deficits. To the extent that damage to the hippocampus and its associated structures is indeed responsible for the path integration deficits we observed, our results support the notion that these structures play a homologous role for path integration in humans and rodents.

\section{METHODS}

\section{Participants}

Three groups of participants gave their informed consent to participate (see Table 2). The experiment was approved by the George Washington University (GWU) Institutional Review Board. Two groups had undergone unilateral MTL resection as therapy for intractable epilepsy, whereas a third (the control group) had no history of neurological disorder. An ANOVA showed no reliable differences in age between groups, $F(2,25)=2.85, p>.05$.

\section{Medial Temporal Lobectomy Procedure}

The surgeries were performed by one of the authors (AJC). This standard procedure consists of a frontaltemporal craniotomy, which exposes the temporal lobe and a small portion of the frontal lobe (Caputy \& Bejjani, 1999). When these cortical surfaces are exposed, an electrocorticography recording array is put into place. The epileptogenic area is mapped and defined, occasionally with the aid of chemical (Brevital $20 \mathrm{mg}$ ) or electrical stimulation. The area of the anterior-lateral temporal lobe to be resected is defined by electrocortographic mapping. Based on this mapping, portions of the anterior and lateral surfaces 
Table 2. Demographic Details and Neuropsychological Performance of the Three Participant Groups ${ }^{\mathrm{a}}$

\begin{tabular}{|c|c|c|c|}
\hline & Healthy Control & Left $T L R^{\mathrm{b}}$ & Right $T L R^{\mathrm{b}}$ \\
\hline $\operatorname{Sex}(M / F)$ & $6 / 4$ & $3 / 5$ & $5 / 5$ \\
\hline Age & $35(20-56)$ & $48(29-59)$ & $42(27-58)$ \\
\hline Handedness (R/L) & $10 / 0$ & $8 / 0$ & $9 / 1$ \\
\hline Time Test ${ }^{\mathrm{c}}$ & N/A & $5.9(3.1-10.8)$ & $3.0(0.6-5.1)$ \\
\hline Wechsler IQ (premorbid) & N/A & $106^{\mathrm{d}}(85-122)$ & $104^{\mathrm{e}}(86-128)$ \\
\hline Rey Copy $(\max =36)$ & $31(28-36)$ & $28(16-34)$ & $28(10-35)$ \\
\hline Rey Delay $(\max =36)$ & $19(4-26)$ & $11(6-20)$ & $14(8-26)$ \\
\hline WMS $\operatorname{LMI}^{\mathrm{g}}(\max =50)$ & $25(5-41)$ & $19(13-27)$ & $19^{\mathrm{f}}(9-25)$ \\
\hline WMS LMII ${ }^{g}(\max =50)$ & $26(1-38)$ & $16(8-23)$ & $18^{\mathrm{f}}(5-28)$ \\
\hline
\end{tabular}

${ }^{a}$ Except where indicated, mean values are presented, with the range in parentheses.

${ }^{\mathrm{b}}$ Temporal lobe resection.

'Time of testing, postsurgery (years).

${ }^{\mathrm{d}} n=7$.

$\mathrm{e}_{n}=6$.

${ }^{\mathrm{f}} n=9$.

gTotal score.

of the temporal lobe are resected until the area is quiescent from an epileptogenic standpoint. The MTL structures are then resected, including the amygdala and anterior portions of the parahippocampal gyrus and hippocampus. The fornix is spared. The temporal lobe resections are measured when resection is determined to be complete from an electrocorticographic standpoint. The measurements are made from the temporal tip in an anterior-rostral to a posteriorcaudal direction. Three centimeters of the hippocampus was removed in all patients. The extent of the temporal lobe lesions was estimated by adding the intraoperative resection measurements along the superior, middle, and inferior temporal gyri. The resulting sums (which yield an approximation of the total lesion area along the cortical surface) averaged 7.9 and $6.4 \mathrm{~cm}$ for the RTLR and LTLR patients, respectively. A twotailed $t$ test performed on these data showed there to be no reliable hemispheric differences, $t(16)=1.21$; $p=.24$. As an additional check, lesion volumes were estimated on the basis of postoperative brain images (computerized tomography). Scans were available for 10 RTLR and 6 LTLR patients. Two neuroradiologists independently estimated, by visual inspection, the percentage of the entire temporal lobe resected. The two readings for each patient differed by no more than $10 \%$ and any discrepancies were averaged. A two-tailed $t$ test performed on these data again showed no hemispheric differences: mean resection percentage $31 \% ; t(14)=1.04 ; p=.31$. Thus, although somewhat less of the lateral temporal lobe was resected in the language-dominant hemisphere, as is common for this procedure, these hemispheric differences were too small to reach statistical significance given the sample size in this study.

The criteria for inclusion included: age 18-75 years, 12 or more years of education, absence of dementia or psychiatric disorder, visual acuity 20/100 or better, no difficulty walking without assistance, rare or no seizures, and left hemisphere language dominance (as assessed by sodium amobarbital testing prior to surgery). All participants were paid a small stipend to help defray transportation and parking costs. The MTL patients all had presurgical intelligence quotients of at least 80 (Wechsler Adult Intelligence Scale-Revised [WAIS-R]) (Wechsler, 1981). Although postsurgical intelligence scores were generally not available, clinical observation suggested that all participants were sufficiently intelligent to meet or exceed a score of 80 on the WAIS-R at the time of testing. A two-tailed $t$ test showed that the groups differed in terms of when testing was conducted relative to the medial temporal lobectomy, $t(16)=-2.67, p=.022$, with the LTLR group being tested somewhat later after surgery.

\section{Neuropsychological Memory Tests}

The performance of the participants on four memory tests were compared in separate ANOVAs: Logical Memory I and II subtests (Total Score measure) of the Wechsler Memory Scale (WMS-III) (Wechsler, 1997) and the copy and delayed recall measures of 
the Rey-Osterreith Complex Figure (ROCF) test. The ROCF test is a common measure of visuospatial memory, involving copying a complex visual figure and then attempting to reproduce it by memory after a 30-min delay. These tests require the retention of information for much longer durations than are involved in the behavioral tasks of the current study, but they are nevertheless standard measures and, therefore, provide a means of comparing our patients with similar patients described elsewhere. Of these tests, only the WMS-III Logical Memory II subtest revealed a significant group difference $(p=.036)$. Pairwise planned contrasts $(p=$ $.05)$ showed that the patient groups both performed significantly more poorly on this test than the control group, but did not differ from each other. One control participant performed quite poorly on most of these tests. When his scores were excluded as outliers, all analyses except for the "copy" measure of the ROCF test showed reliable group effects $(p<.05)$, with pairwise planned contrasts $(p=.05)$ again showing that the two patient groups performed similarly and both performed more poorly than the control group. In the "copy" test, the participant can see the sample figure while copying it, so the lack of a group effect in this test suggests that all groups were approximately equated in terms of visuospatial attention.

\section{Design and Apparatus}

The experiment took place in a well-lit classroom. Each observer participated in four blocked conditions presented in the following order: target-directed walking, experimenter-guided walking, verbal distance estimation, and delayed distance matching. The order of trials within each of these blocks was randomized. In the two walking conditions, stimulus distances of 2.5 and $5 \mathrm{~m}$ were presented five times apiece. In targetdirected trials, the stimulus was a $23-\mathrm{cm}$ tall cone; in experimenter-guided trials, an experimenter presented the stimulus distance by guiding the blindfolded participant along a linear path of the appropriate length. To discourage pace-counting strategies, slow and fast walking paces (approximately 1 and $2 \mathrm{~m} / \mathrm{sec}$, respectively) were each imposed on two of the five measurements of each stimulus distance, with a medium pace (approximately $1.5 \mathrm{~m} / \mathrm{sec}$ ) imposed on the fifth. Pace length increases with walking speed (Mittelstaedt \& Mittelstaedt, 2001), so this manipulation makes pace counting an unreliable strategy. This distribution of walking speeds ensured that such an effect would not systematically bias the overall pattern of responses. To provide a greater range of distances, stimulus distances of $2,3,4$, and $6 \mathrm{~m}$ were presented twice apiece. These distances were randomly paired with the slow and fast walking paces for each participant. Because these distances were not systematically crossed with walking pace, we did not subsequently analyze these trials. In verbal distance estimation and delayed distance matching trials, the stimulus cone was presented at distances of $2,2.5,3,4,5$, and $6 \mathrm{~m}$ three times apiece in random order.

\section{Procedure}

\section{Target-Directed Walking}

Participants viewed the target cone, then lowered a blindfold and attempted to walk to the target's location while holding onto the experimenter's arm for support. An assistant removed the target before walking began, and the experimenter kept his or her eyes closed until the target was removed so as to remain blind to the target distance. Participants were instructed not to use a pace-counting strategy. While walking with the participant, the experimenter imposed one of three possible walking speeds on each trial. This prevented veering and ensured consistency across participants in terms of response durations. After responding, participants were led back to the starting location without vision and without error feedback. The straight-line walked distance was recorded via tape measure.

\section{Experimenter-Guided Walking}

Participants viewed the environment for several seconds (without a specified target). They then lowered the blindfold and grasped the experimenter's arm. The experimenter walked along a straight path with a length of 2 to $6 \mathrm{~m}$, using one of the three possible walking speeds. At the end of the path, the participants verbally estimated the distance walked from the origin. They were then guided back to the starting position without error feedback. The stimulus distance imposed by the experimenter tended to vary slightly from the nominal distances; these variations were recorded and the statistical analyses were conducted on the response errors relative to the stimulus distance actually presented on each trial.

\section{Verbal Distance Estimation}

Participants viewed the target and verbally estimated its distance from their feet.

\section{Delayed Distance Matching}

Participants viewed a stimulus cone (the standard) in the laboratory while standing in the laboratory doorway; they then walked $1.5 \mathrm{~m}$ to a second viewing location in an adjoining hallway to see an identical comparison cone. The standard was not visible from the second viewpoint. At the beginning of each trial, the comparison cone was placed $6.5 \mathrm{~m}$ from the observer's position at the second viewpoint. Five seconds after the participant reached the second viewpoint, an experi- 
menter began to move the cone towards the participant. The participant directed the experimenter to move the comparison cone until its distance from the participant's toes matched the remembered distance of the standard cone as seen from the first viewpoint. This task imposes far fewer demands on visuospatial memory than does the ROCF test, but is much more appropriate for assessing the memory of a target location long enough to walk to it without vision.

\section{Acknowledgments}

A preliminary report of this work was presented in April, 2002 at the annual meeting of the Cognitive Neuroscience Society in San Francisco, CA. This work was supported in part by a grant from the National Institutes of Mental Health (NIMH 54216) to Marlene Behrmann. The authors thank Kurt Richer, Steven Brown, Sameer Pandit, J'aime Hall, Kamil Barker and Jonathan Brigman for their assistance in conducting the experiment.

Reprint requests should be sent to John W. Philbeck, Department of Psychology, The George Washington University, 2125 G. Street, NW, Washington, DC 20052, or via e-mail: philbeck@gwu.edu.

\section{REFERENCES}

Aguirre, G. K., Detre, J. A., Alsop, D. C., \& D'Esposito, M. (1996). The parahippocampus subserves topographical learning in man. Cerebral Cortex, 6, 823-829.

Astur, R. S., Taylor, L. B., Mamelak, A. N., Philpott, L., \& Sutherland, R. J. (2002). Humans with hippocampus damage display severe spatial memory impairments in a virtual Morris water task. Behavioural Brain Research, 132, 77-84.

Bohbot, V. D., Kalina, M., Stepankova, K., Spackova, N., Petrides, M., \& Nadel, L. (1998). Spatial memory deficits in patients with lesions to the right hippocampus and to the right parahippocampal cortex. Neuropsychologia, 36, 1217-1238

Burgess, N., Maguire, E. A., \& O'Keefe, J. (2002). The human hippocampus and spatial and episodic memory. Neuron, 35, 625-641.

Caputy, A., \& Bejjani, G. (1999). Surgical treatment of intractable epilepsy. In L. N. Sekhar \& E. D. Oliveira (Eds.), Cranial microsurgery. Approaches and techniques. (pp. 278-292). New York: Thieme.

Eichenbaum, H. B., \& Cohen, N. J. (2001). From conditioning to conscious recollection: Memory systems of the brain. Oxford: Oxford University Press.

Etienne, A. S., Maurer, R., \& Séguinot, V. (1996). Path integration in mammals and its interaction with visual landmarks. Journal of Experimental Biology, 199, 201-209.

Feigenbaum, J. D., Polkey, C. E., \& Morris, R. G. (1996). Deficits in spatial working memory after unilateral temporal lobectomy in man. Neuropsychologia, 34, 163-176.

Grön, G., Wunderlich, A. P., Spitzer, M., Tomczak, R., \& Riepe, M. W. (2000). Brain activation during human navigation: Gender-different neural networks as subtrate of performance. Nature Neuroscience, 3, 404-408.

Hamilton, D. A., Driscoll, I., \& Sutherland, R. J. (2002). Human place learning in a virtual Morris water task: Some important constraints on the flexibility of place navigation. Behavioural Brain Research, 129, 159-170.

Klatzky, R. L., Beall, A. C., Loomis, J. M., Golledge, R. G., \& Philbeck, J. W. (1999). Human navigation ability: Tests of the encoding-error model of path integration. Spatial Cognition and Computation, 1, 31-65.

Logothetis, N. K., \& Sheinberg, D. L. (1996). Visual object recognition. Annual Review of Neuroscience, 19, 577-621.

Maguire, E. A., Burgess, N., Donnett, J. G., Frackowiak, R. S. J., Frith, C. D., \& O'Keefe, J. (1998). Knowing where and getting there: A human navigation network. Science, 280, 921-924.

Markus, E. J., Barnes, C. A., McNaughton, B. L., Gladden, V. L., \& Skaggs, W. E. (1994). Spatial information content and reliability of hippocampal CA1 neurons: Effects of visual input. Hippocampus, 4, 410-421.

Mittelstaedt, M.-L., \& Mittelstaedt, H. (2001). Idiothetic navigation in humans: Estimation of path length. Experimental Brain Research, 139, 318-332.

Morris, R. G. M., Garrud, P., Rawlins, J. N. P., \& O'Keefe, J. (1982). Place navigation impaired in rats with hippocampal lesions. Nature, 297, 681-683.

Murray, E. A., \& Mishkin, M. (1998). Object recognition and location memory in monkeys with excitotoxic lesions of the amygdala and hippocampus. Journal of Neuroscience, 18, 6568-6582.

Nunn, J. A., Graydon, F. J., Polkey, C. E., \& Morris, R. G. (1999). Differential spatial memory impairment after right temporal lobectomy demonstrated using temporal titration. Brain, 122, 47-59.

O'Keefe, J., \& Dostrovsky, J. (1971). The hippocampus as a spatial map. Preliminary evidence from unit activity in the freely-moving rat. Brain Research, 34, 171-175.

O'Mara, S. M., Rolls, E. T., Berthoz, A., \& Kesner, R. P. (1994). Neurons responding to whole-body motion in the primate hippocampus. Journal of Neuroscience, 14, 6511-6523.

Owen, A. M., Milner, B., Petrides, M., \& Evans, A. C. (1996). A specific role for the right parahippocampal gyrus in the retrieval of object-location: A positron emission tomography study. Journal of Cognitive Neuroscience, 8, 588-602.

Philbeck, J. W., Klatzky, R. K., Behrmann, M., Loomis, J. M., \& Goodridge, J. (2001). Active control of locomotion facilitates nonvisual navigation. Journal of Experimental Psychology: Human Perception and Performance, 27, 141-153.

Philbeck, J. W., \& Loomis, J. M. (1997). Comparison of two indicators of perceived egocentric distance under full-cue and reduced-cue conditions. Journal of Experimental Psychology: Human Perception and Performance, 23, 72-85.

Pierrot-Deseilligny, C., Müri, R. M., Rivaud-Pechoux, S., Gaymard, B., \& Ploner, C. J. (2002). Cortical control of spatial memory in humans: The visuooculomotor model. Annals of Neurology, 52, 10-19.

Quirk, G. J., Muller, R. U., \& Kubie, J. L. (1990). The firing of hippocampal place cells in the dark depends on the rat's recent experience. Journal of Neuroscience, 10, 2008-2017.

Redish, A. D. (1999). Beyond the cognitive map: From place cells to episodic memory. Cambridge: MIT Press.

Redish, A. D. (2001). The hippocampal debate: Are we asking the right questions? Behavioural Brain Research, 127, 81-98.

Skelton, R. W., Bukash, C. M., Laurance, H. E., Thomas, K. G. F., \& Jacobs, W. J. (2000). Humans with traumatic brain injuries show place-learning deficits in computer-generated virtual space. Journal of Clinical and Experimental Neuropsychology, 22, 157-175.

Smith, M. L., \& Milner, B. (1981). The role of the right hippocampus in the recall of spatial information. Neuropsychologia, 19, 781-793. 
Spiers, H. J., Burgess, N., Maguire, E. A., Baxendale, S. A., Hartley, T., Thompson, P. J., \& O'Keefe, J. (2001).

Unilateral temporal lobectomy patients show lateralized topographical and episodic memory deficits in a virtual town. Brain, 124, 2476-2489.

Squire, L. R. (1992). Memory and the hippocampus: A synthesis from findings with rats, monkeys, and humans. Psychological Review, 99, 195-231.

Sutherland, R. J., \& McDonald, R. J. (1990). Hippocampus, amygdala, and memory deficits in rats. Behavioural Brain Research, 37, 57-79.

Vargha-Khadem, F., Gadian, D. G., Watkins, K. E., Connelly, A., Van Paesschen, W., \& Mishkin, M. (1997). Differential effects of early hippocampal pathology on episodic and semantic memory. Science, 277, 376-380.

Wang, R. F., \& Spelke, E. S. (2002). Human spatial representation: Insights from animals. Trends in Cognitive Sciences, 6, 376-382.

Wechsler, D. (1981). Wechsler Adult Intelligence Scale Revised. New York: The Psychological Corporation.
Wechsler, D. (1997). Wechsler Memory Scale-third edition. San Antonio: The Psychological Corporation.

Wehner, R., Michel, B., \& Antonsen, P. (1996). Visual navigation in insects: Coupling of egocentric and geocentric information. Journal of Experimental Biology, 199, 129-140.

Whishaw, I. Q., Hines, D. J., \& Wallace, D. G. (2001). Dead reckoning (path integration) requires the hippocampal formation: Evidence from spontaneous exploration and spatial learning tasks in light (allothetic) and dark (idiothetic) tests. Behavioural Brain Research, 127, 49-69.

Wiest, G., Müller, C., Glück, J., Deecke, L., \& Baumgartner, C. (2000). Idiothetic orientation and path integration in unilateral hippocampal atrophy and sclerosis. Journal of Vestibular Research, 10, 301-309.

Worsley, C. L., Recce, M., Spiers, H. J., Marley, J., Polkey, C. E., \& Morris, R. G. (2001). Path integration following temporal lobectomy in humans. Neuropsychologia, 39, 452-464. 
Copyright of Journal of Cognitive Neuroscience is the property of MIT Press and its content may not be copied or emailed to multiple sites or posted to a listserv without the copyright holder's express written permission. However, users may print, download, or email articles for individual use. 
Copyright of Journal of Cognitive Neuroscience is the property of MIT Press and its content may not be copied or emailed to multiple sites or posted to a listserv without the copyright holder's express written permission. However, users may print, download, or email articles for individual use. 